\title{
Factores que influyen en el abandono temprano de la lactancia por mujeres trabajadoras
}

\author{
Manuel N avarro-Estrella, MC, ${ }^{(1)}$ María Ximena Duque-López, $M$ en $C_{1}{ }^{(2)}$ \\ Juan Antonio Trejo y Pérez, MC..$^{(3)}$
}

\section{Navarro-Estrella M, Duque-López MX,Trejo y Pérez JA. Factores que influyen en el abandono temprano de la lactancia por mujeres trabajadoras. Salud Publica Mex 2003;45:276-284.} El texto completo en inglés de este artículo está disponible en: http://www.insp.mx/salud/index.html

\begin{abstract}
Resumen
Objetivo. Identificar los factores maternos, laborales y de los servicios de salud que influyen en el abandono temprano de la lactancia materna en madres trabajadoras. Ma terial y métodos. Entre noviembre de 1998 y marzo de 1999 se efectuó un estudio transversal comparativo con madres derechohabientes del Instituto Mexicano del Seguro Social de Ensenada, Baja California, México.A 265 madres se les aplicó un cuestionario entre los tres y nueve meses posparto. Se distribuyeron en: grupo I: madres con abandono temprano de la lactancia materna; grupo II: madres que prolongaron la lactancia materna por más de tres meses. Para identificar los factores asociados con el abandono temprano de la lactancia materna, se utilizó regresión logística. Resultados. El 42.3\% (112) de las madres abandonaron temprano la lactancia materna. Los factores de riesgo fueron: tener conocimientos malos sobre lactancia materna, O R 5.97 (IC 95\% 1.67-20.67), la ausencia del antecedente de haberla practicado en un hijo previo OR 2.98 (IC 95\% 1.66-5.36), tener un plan de duración de la misma de 0 a 3 meses, O R 16.24 (IC 95\% 5.37-49.12), y la falta de facilidades en el trabajo para efectuarla, OR 1.99 (IC 95\% 1.12-3.56). Conclusiones Los principales factores asociados con el abandono temprano de la lactancia materna fueron maternos. El único factor laboral fue la ausencia de
\end{abstract}

\author{
Navarro-Estrella M, Duque-López MX,Trejo y Pérez JA. \\ Factors associated with short duration of breast-feeding \\ in Mexican working women. \\ Salud Publica Mex 2003;45:276-284. \\ The English version of this paper \\ is available at: http://www.insp.mx/salud/index.html
}

\begin{abstract}
A bstract
Objective.To identify the maternal, work, and health services factors associated with a short duration of breast-feeding in working mothers. Material and Methods. A cross-sectional study was carried out between N ovember 1998 and March 1999, among 265 mothers medically insured by (Instituto Mexicano del Seguro Social, IMSS) Mexican Institute of Social Security, who completed a questionnaire when their babies were 3 to 9 months old. Mothers were divided into two groups, based on breast-feeding duration: those who breast-fed for less than 3 months and those who did so for three months or more. Logistic regression was used to identify the factors associated with short duration of breast-feeding. Results. 0 ne-hundred and twelve mothers (42.3\%) abandoned breast-feeding early. Risk factors were:poor maternal knowledge on breast-feeding, odds ratio (O R) 5.97,95\% confidence interval (CI) 1.67-20.67; mothers with no previous breast-feeding experience OR $2.98,95 \%$ $\mathrm{Cl}$ 1.66-5.36; mothers planning to breast-feed their babies for less than 3 months (O R 16.24, 95\% CI 5.37-49.12); and mothers with no access to breast-feeding facilities at work (O R $1.99,95 \% \mathrm{Cl} 1.12-3.56)$. Conclusions. The main factors associated with short duration of breast-feeding were the maternal ones.The only work-related factor associated with short duration of breast-feeding was the absence of
\end{abstract}

(1) Unidad de Medicina Familiar número 25, Instituto Mexicano del Seguro Social (IMSS), Ensenada, Baja California, México.

(2) Unidad de Investigación en Epidemiología N utricional. División de Investigación Epidemiológica y en Servicios de Salud. Coordinación de Investigación en Salud, IMSS, México, D F, México.

(3) Unidad de Investigación Epidemiológica y en Servicios de Salud. División de Investigación Epidemiológica y en Servicios de Salud. Coordinación de Investigación en Salud, IMSS, México, DF, México.

Fecha de recibido: 20 de mayo de 2002 • Fecha de aprobado: 15 de abril de 2003

Solicitud de sobretiros: María Ximena D uque L. Unidad de Investigación en Epidemiología N utricional. 3er. Piso del edificio anexo a la farmacia. Centro Médico N acional Siglo XXI.Avenida Cuauhtémoc N 0. 330, Colonia D octores, 06725 México, DF, México.

Correo electrónico:xduque@ prodigy.net.mx 
facilidades para amamantar. Es probable que la calidad de los conocimientos, la experiencia previa con ella y tener facilidades laborales influyan en la decisión de prolongarla. El texto completo en inglés de este artículo está disponible en: http://www.insp.mx/salud/index.html

Palabras clave: abando no de lactancia materna; mujeres trabajadoras; factores de riesgo; México breast-feeding facilities. It is pro bable that maternal knowledge on breast-feeding, previous breast-feeding experience, and the availability of facilities at work for breast-feeding affect the maternal decisions on breast-feeding duration.The English version of this paper is available at: http:// www.insp.mx/salud/index.html

Key words: short breast-feeding; working mothers; risk factors; Mexico a leche humana posee cualidades que producen beneficios significativos para la madre y su hijo. Entre éstos destacan los nutricios, los inmunológicos y los afectivos. En países en desarrollo como México el aspecto económico adquiere también un lugar preponderante. ${ }^{1,2}$

En contraste con las ventajas de la lactancia materna (LM) es notable la disminución creciente de su práctica en países en desarrollo, tanto en áreas urbanas como en las rurales. ${ }^{3-8}$ Los factores que se han asociado con su abandono son diversos y difieren de acuerdo con el sitio de residencia, las características socioeconómicas y culturales. ${ }^{9}$ En América Latina es notorio que un mejor nivel socioeconómico, una mayor escolaridad materna y el trabajo fuera del hogar se asocian con porcentajes mayores de abandono temprano de esta práctica. ${ }^{10-15}$

Al respecto, en nuestro país en los últimos 30 años se ha sufrido un deterioro importante, caracterizado por una disminución progresiva de la LM exclusiva, la reducción a menos de $50 \%$ de la lactancia parcial a los tres meses de edad, y una introducción cada día más temprana de los sucedáneos de la leche materna. En la región norte de México estas características se dan con mayor frecuencia que en el sur y centro del país. ${ }^{8,16}$

Los escenarios descritos han conducido a la Organización Mundial de la Salud (OMS) y al Fondo de las Naciones Unidas para la Infancia (UNICEF) a promover compromisos internacionales y al establecimiento de actividades como el alojamiento conjunto de la madre y el neonato y, en 1989, de programas hospitalarios durante la atención del parto, como el del Hospital Amigo del Niño y de la Madre (PHANM), en el cual se alientan el inicio temprano y exclusivo de la LM, se asiste a la madre en el procedimiento del amamantamiento y se proscribe la utilización de biberones y sucedáneos de la leche materna. ${ }^{17-20}$ En Bielorusia se ha demostrado recientemente que el programa incrementa la duración de la $\mathrm{LM}^{21}$ y el UNICEF promueve que se lleven a cabo evaluaciones del impacto y funcionali- dad del programa en todos los hospitales donde la estrategia está implantada, lo anterior con base en que las diferencias locales en recursos materiales y humanos pueden conducir a diferencias en la efectividad. ${ }^{19}$

En México, un estudio realizado en una muestra representativa de los servicios de salud de Chiapas, Sonora y Ciudad de México, entre 1989 y 1991, reportó un cumplimiento muy bajo de las recomendaciones dadas por UNICEF/OMS y de La Ley General de Salud Mexicana, en lo referente a la atención de los recién nacidos y, por el contrario, lejos de incentivar la LM se muestra un decremento en la expectativa de alimentar exclusivamente con leche materna entre el ingreso hospitalario y el egreso posparto, dando alimentación al recién nacido con soluciones glucosadas y con sucedáneos de leche materna y sin el alojamiento conjunto ${ }^{20}$ hallazgos que demuestran los retos a los que se enfrentaría la aplicación del PHANM.

En Ensenada, Baja California, la participación económica de la mujer es creciente y actualmente más de $40 \%$ de la población femenina en edad fértil realiza actividades remuneradas, ${ }^{22}$ sus hijos son atendidos en guarderías donde se les alimenta con sucedáneos de la leche materna (SLM) hasta los cuatro meses de edad, y posteriormente, con fórmula de continuación en biberón; lo anterior se cumple en el escenario de la operación del programa Hospital Amigo del Niño, desde 1994. Por lo que se ha descrito es nuestro interés identificar los factores maternos, de los servicios de salud y laborales que influyen en el abandono temprano de la LM en esta localidad, con la intención de identificar aquellos susceptibles de ser modificados.

\section{Material y métodos}

Se realizó un estudio transversal comparativo en Ensenada, Baja California, México, en la población derechohabiente de madres trabajadoras atendidas en el Hospital General de Zona IV No. 8, del Instituto Mexicano del Seguro Social (IMSS), cuyos hijos nacieron entre noviembre de 1998 y marzo de 1999. Este hospi- 
tal atiende todos los nacimientos de la población asegurada de la ciudad. Se incluyeron en el estudio a todas las madres sanas con productos únicos, también sanos, con edad gestacional igual o mayor a 37 semanas, nacidos en el periodo mencionado. El estudio fue aprobado por los comités de ética e investigación correspondientes.

\section{Variables de estudio}

a) Variable dependiente. Abandono temprano de la LM. Se consideró esta condición cuando la leche materna fue retirada de la alimentación del niño a los tres meses de edad o antes. Los 3, 6 y 9 meses de edad son valores de corte que han sido ampliamente utilizados para describir la frecuencia de la LM, y para evaluar los efectos de la misma sobre la morbilidad y los factores asociados a su duración. ${ }^{9,20,21,23,24}$

b) Las variables independientes se distribuyeron en: factores maternos (edad, escolaridad, estado civil, existencia de red de apoyo familiar, conocimientos sobre LM, antecedente de LM en un hijo previo, decisión de dar leche materna y tiempo planeado para la duración de la LM). Factores laborales (tipo de ocupación, duración de la jornada de trabajo y facilidades dadas en éste para la LM) y factores de los servicios de salud (control prenatal, trimestre durante el cual se tuvo la primera consulta y número de consultas; tipo de nacimiento, haber recibido la demostración para la LM, inicio de la alimentación con leche materna en el hospital, alojamiento conjunto y red de apoyo hospitalaria).

Las variables de estudio se obtuvieron con la aplicación a las madres seleccionadas de un cuestionario estandarizado. Se llevó a cabo la capacitación previa de las trabajadoras sociales para la realización de la encuesta y el control de calidad de esta última. La aplicación del cuestionario en la mayoría de los casos fue en el domicilio particular; cuando esto no fue posible, la señora se entrevistó en su trabajo. La encuesta se realizó entre el 15 de abril y el 30 de septiembre de 1999.

\section{Definiciones principales}

Se consideró lactancia materna casi exclusiva (LMCE) cuando la alimentación del niño fue únicamente con leche materna, complementada o no con infusiones de yerbas y agua.
La lactancia mixta se refiere a la alimentación al pecho materno complementada con SLM en menores de cuatro meses de edad y fórmula de seguimiento en mayores de esta edad.

Los patrones de lactancia materna se obtuvieron mediante recordatorio histórico de alimentación del niño, desde su nacimiento hasta el momento de la entrevista. La mediana de la edad de los niños cuando se realizó la entrevista fue de 6 (3 - 9) meses.

La calificación de los conocimientos sobre LM se dio con base en la identificación de cuatro contenidos en las respuestas maternas sobre las ventajas de la alimentación con leche materna, y las características de su administración:

1. Menos infecciones en el niño

2. El desarrollo del vínculo entre madre e hijo

3. Lo innecesario de complementar a la LM con infusiones de yerbas o agua

4. Que el mejor horario de alimentación con leche materna es el que se acoge a libre demanda

A los cuatro contenidos se les asignó un valor equivalente de uno, si estaba presente, o de cero, en su ausencia; la identificación de los cuatro contenidos fue igual a conocimientos buenos; dos o tres, igual a regulares y cero a uno, igual a conocimientos malos.

\section{A nálisis}

Para comparar la duración de los diferentes patrones de lactancia entre los dos grupos se utilizó $\chi^{2}$. En la comparación de las proporciones entre los dos grupos en las diferentes variables estudiadas se utilizaron las pruebas de $\chi^{2}$, exacta de Fisher, $U$ de Mann-Whitney y prueba $t$. Para identificar los factores asociados con el abandono temprano de la LM se utilizó análisis multivariado por regresión logística, incluyendo en el modelo aquellas variables que en el análisis univariado mostraron un valor de $p$ menor o igual a 0.20 ; se evaluaron variables potencialmente confusoras e interacciones. Para evaluar el modelo se obtuvo el estadístico de Hosmer-Lemeshow, y se hizo una tabla de clasificación, determinando la sensibilidad, especificidad y el porcentaje de observaciones correctamente clasificadas. ${ }^{25} \mathrm{El}$ análisis se realizó utilizando el programa Stata versión 6.0. ${ }^{26}$

\section{Resultados}

Participaron en el estudio 265 madres trabajadoras derechohabientes del IMSS, cuya entrevista se llevó a 
cabo entre el 15 de abril y el 30 de septiembre de 1999. Al momento de la aplicación del cuestionario sus hijos tenían entre 3 y 9 meses de edad.

El $42 \%$ de estas madres (112/265) abandonaron totalmente la LM a los tres meses de edad de sus hijos o antes, y con ellas se integró el grupo I. El 11.6\% de estas madres nunca dio ni lactancia materna exclusiva ni casi exclusiva a sus hijos, y la mediana de edad a la que iniciaron SLM fue a los 28.5 ( 1 a 91) días, mientras que las madres del grupo II (153/265), quienes alimentaron a sus hijos con leche materna por más de tres meses, no dieron LME ni LMCE sólo en $4.6 \%$ de los casos, e iniciaron la fórmula a los 61 días (1 a 226), $(p<0.001$ para ambas características en la comparación entre grupos). La mediana de la edad a la que se realizó el destete en el grupo I fue 45 ( 1 a 90) días y en el grupo II fue 142 (105 a 255) días $(p<0.001)$. Las principales razones para el destete reportadas por las madres del grupo I fueron: el trabajo (25.9\%), producción insuficiente de leche (20.5\%) y el rechazo por parte del niño (15.2\%). En el grupo II, las principales razones fueron: el trabajo (16.4\%), y la producción insuficiente $(9.2 \%)$. Todas las madres participantes retornaron a sus actividades laborales en promedio a los $45( \pm 7)$ días posparto.

En la figura 1 se muestra la duración de la LMCE y el inicio del SLM; la duración de la LMCE es me-

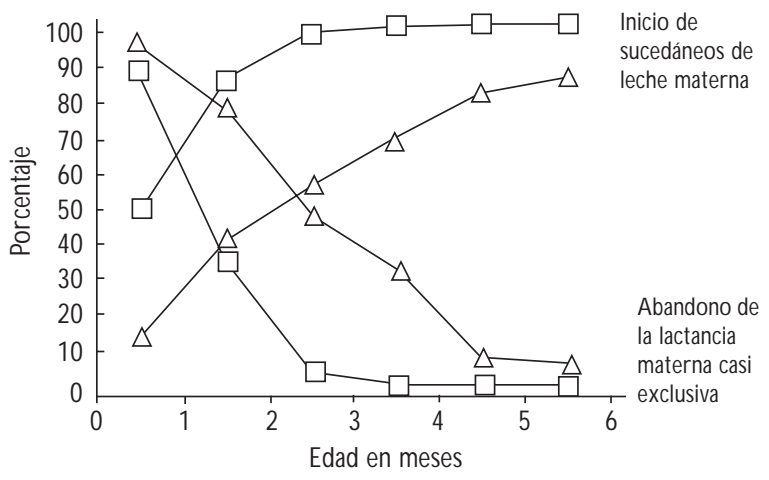

Grupo I:madres que abandonaron la lactancia materna a los tres meses 0 antes

$\triangle$ Grupo II: madres que lactaron por más de tres meses

$p<0.05$ entre los grupos en cada mes, para abandono de la lactancia materna casi exclusiva y para inicio de sucedáneos de leche materna

Figura 1. Abandono de la lactancia materna casi EXCLUSIVA E INICIO DE SUCEDÁNEOS DE LECHE MATERNA, EN DOS GRUPOS DE MADRES TRABAJAdORAS. ENSENADA, Baja California, México, 1998-1999 nor y el cambio en su proporción mayor en el grupo I; puede observarse que en este grupo la LMCE disminuyó a $34.8 \%$ en el segundo mes de vida, mientras que en el grupo II se observa un porcentaje semejante $(32.0 \%)$ hasta el cuarto mes de edad. De igual forma, el tiempo al cual se inicia SLM en el grupo I es significativamente más temprano que en el grupo II $(p<0.05)$, mientras que en el grupo I todas las madres habían abandonado por completo la LM e iniciado SLM como único alimento lácteo a los tres meses posparto; en el grupo II $53.6 \%$ de las madres abandonó la LMCE a esta edad, pero prolongaron la LM por medio de la lactancia mixta, e incluso $19.0 \%$ de las madres no habían iniciado SLM a los cinco meses de edad.

En el cuadro I se muestran los factores maternos en relación con el abandono temprano de la LM. La edad promedio de las madres, la proporción de ellas en cada grupo de escolaridad y del estado civil fueron semejantes tanto en el grupo con abandono temprano de la LM como en aquel que la prolongó más allá de los tres meses posparto ( $p>$ de 0.200 ). En relación con los conocimientos maternos sobre LM, la proporción de madres con conocimientos malos fue mayor en el grupo I ( $26.8 \%$ vs $14.4 \%$ ) y la proporción de éstas con conocimientos buenos fue mayor en el grupo II (15.7 vs 3.6\%), $(p=0.001)$. En contraste, el número de madres con el antecedente de haber experimentado la LM en un hijo previo, y el de aquellas con un plan de administración de LM durante más de seis meses fue mayor en el grupo II ( $p \leq 0.002$ para los dos factores entre ambos grupos).

En el cuadro II se pueden ver los factores relacionados con los servicios de salud y con la promoción de la LM en tres niveles de apoyo; la atención prenatal, la atención hospitalaria y, finalmente, la asesoría que puede ser lograda a partir de redes de apoyo, como serían los grupos de autoayuda, coordinados por el hospital, y aquella que puede obtenerse de familiares o amistades cercanas. El $80 \%$ o más de las madres de ambos grupos tuvieron su primera consulta de control prenatal durante el primer trimestre de la gestación, y $75 \%$ o más de ellas tuvieron al menos siete consultas antes de la resolución de su embarazo; todas las madres participantes en este estudio recibieron información sobre las ventajas de la lactancia materna durante la atención prenatal. En la atención hospitalaria la proporción de madres que concluyeron su embarazo por parto vaginal o cesárea fue semejante en ambos grupos ( $p=0.294)$. En cuanto a las acciones de asistencia para la LM dentro del hospital y el inicio temprano de la experiencia de lactar, $80 \%$ o más de las madres de los grupos recibieron asesoría sobre la técnica adecuada para la lactancia; asimismo, en más de 


\begin{tabular}{|c|c|c|c|c|c|}
\hline \multicolumn{6}{|c|}{ Cuadro I } \\
\hline \multirow{3}{*}{\multicolumn{6}{|c|}{$\begin{array}{l}\text { ABANDONO TEMPRANO DE LA LACTANCIA MATERNA } \\
\text { EN RELACIÓN CON LOS FACTORES MATERnOS. } \\
\text { ENSENADA, BAJA CALIFORNIA, MÉXICO, 1998-1999 }\end{array}$}} \\
\hline & & & & & \\
\hline & & & & & \\
\hline \multirow[b]{3}{*}{ Factor } & \multicolumn{4}{|c|}{ Lactancia materna } & \multirow[b]{3}{*}{ Valor $p$} \\
\hline & \multicolumn{2}{|c|}{$\begin{array}{c}\leq 3 \text { meses } \\
112(42.3 \%)\end{array}$} & \multicolumn{2}{|c|}{$\begin{array}{l}>3 \text { meses } \\
153(57.7 \%)\end{array}$} & \\
\hline & $\mathrm{n}$ & $\%$ & $n$ & $\%$ & \\
\hline Edad promedio* & \multicolumn{2}{|c|}{$26.1 \pm 05.7$} & \multicolumn{2}{|c|}{$26.0 \pm 05.4$} & 0.270 \\
\hline \multicolumn{6}{|l|}{ Escolaridad } \\
\hline Primaria & 54 & 48.2 & 73 & 47.7 & \\
\hline Secundaria & 25 & 22.3 & 42 & 27.4 & \\
\hline Preparatoria o mayor & 33 & 29.5 & 38 & 24.8 & 0.551 \\
\hline \multicolumn{6}{|l|}{ Estado civil } \\
\hline Soltera & 33 & 29.5 & 32 & 20.9 & \\
\hline Casada & 56 & 50.0 & 88 & 57.5 & \\
\hline Unión libre & 23 & 20.5 & 33 & 21.6 & 0.268 \\
\hline \multicolumn{6}{|c|}{ Conocimientos sobre lactancia materna } \\
\hline Buenos & 4 & 3.6 & 24 & 15.7 & \\
\hline Regulares & 78 & 69.6 & 107 & 69.9 & \\
\hline Malos & 30 & 26.8 & 22 & 14.4 & 0.001 \\
\hline \multicolumn{6}{|c|}{ Lactancia materna en un hijo previo } \\
\hline Sí & 38 & 33.9 & 85 & 55.6 & \\
\hline No & 74 & 66.1 & 68 & 44.4 & 0.002 \\
\hline \multicolumn{6}{|c|}{ Decisión de dar lactancia materna } \\
\hline No & 6 & 5.4 & 1 & 0.6 & \\
\hline Sí & 106 & 94.6 & 152 & 99.4 & 0.018 \\
\hline
\end{tabular}

Plan de duración de la lactancia materna ${ }^{\ddagger}$

\begin{tabular}{lrrrrr} 
De 0 a 3 meses & 27 & 25.5 & 6 & 4.0 & \\
\hline De 4 a 6 meses & 55 & 51.9 & 83 & 54.6 & \\
\hline Siete 0 más & 17 & 16.0 & 55 & 36.2 & \\
\hline Sin plan preciso & 7 & 6.6 & 8 & $5.3<0.001$ \\
$\begin{array}{l}\text { * Media } \pm \text { desviación estándar } \\
\text { ₹ } n=258, \text { se excluyó a siete madres que manifestaron no planear dar le- } \\
\text { che materna a sus hijos }\end{array}$
\end{tabular}

92\% de los casos se realizó la primera tetada en el hospital, evento que sucedió dentro de las primeras dos horas posparto en $60 \%$ o más de las madres. En relación con las redes de apoyo sólo se invitó a $21.4 \%$ de las madres del grupo I y a $29.4 \%$ de las del grupo II a asistir a grupos de autoayuda para apoyo a la LM; lo anterior se suma a que $41.0 \%$ de las madres del grupo I y $44.4 \%$ de las del grupo II no buscó asesoría cuando tuvo alguna duda o problema derivado del ejercicio de la LM.

\section{Cuadro II \\ Abandono temprano de la lactancia materna EN RELACIÓN CON EL SERVICIO DE SALUd. Ensenada, Baja California, México, 1998-1999 \\ Factor

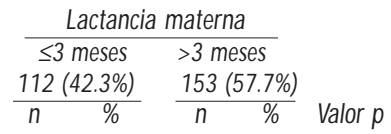

Control prenatal (Sí)

Inicio en el primer trimestre

\begin{tabular}{llllll} 
de la gestación & 92 & 82.1 & 121 & 80.1 & 0.194 \\
\hline
\end{tabular}

$\begin{array}{lllllll}\text { Tuvo siete } 0 \text { más consultas } & 84 & 75.0 & 116 & 76.8 & 0.732\end{array}$

Tipo de nacimiento

\begin{tabular}{llllll} 
Parto normal & 73 & 65.2 & 90 & 58.8 & \\
\hline Cesárea & 39 & 34.8 & 63 & 41.2 & 0.294
\end{tabular}

Le mostraron cómo darle LM

$\begin{array}{llllll}\text { a su hijo (Sí) } & 96 & 85.7 & 122 & 79.7 & 0.208\end{array}$

Dio por primera vez LM en

$\begin{array}{llllll}\text { el hospital (Sí) } & 104 & 92.9 & 144 & 94.1 & 0.679\end{array}$

Dio LM entre las dos primeras

\begin{tabular}{lrrrrr} 
horas posparto (Sí) & 67 & 59.8 & 102 & 66.7 & 0.292 \\
\hline Parto & 51 & 76.1 & 65 & 63.7 & \\
\hline Cesárea & 16 & 23.9 & 37 & 36.3 & 0.089
\end{tabular}

La invitaron a asistir a un grupo

de apoyo a la LM (Sí) $\quad \begin{array}{llllll}24 & 21.4 & 45 & 29.4 & 0.144\end{array}$

Buscó consejo sobre alguna duda

o problema sobre la LM con:

Mamá, hermana, tía, amiga

\begin{tabular}{lrrrrr}
0 suegra & 61 & 54.5 & 74 & 48.4 & \\
\hline Personal de salud & 5 & 4.5 & 11 & 7.2 & \\
\hline Nadie & 46 & 41.0 & 68 & 44.4 & 0.487
\end{tabular}

LM = Lactancia materna

Los factores laborales son expuestos en el cuadro III. Destacan en ambos grupos que aproximadamente la mitad de las madres se ocupan en trabajos que demandan un rendimiento físico elevado, como son: maquiladora, obrera, cocinera y servicios de limpieza; de igual manera, más de $80 \%$ de ellas laboran en jornadas de ocho o más horas de duración. El factor que muestra una distribución diferente es el de contar con facilidades para la LM en el trabajo, predominando en las madres que prolongaron ésta más allá de los tres meses posparto $(52.9 \%$ vs $36.6 \%$, $p=0.008)$. La facilidad que se reportó más frecuentemente fue la hora de lac- 
tancia, lo que significa una hora menos de labores, aplicada al principio o al final de la jornada de trabajo.

En el análisis multivariado (cuadro IV) los principales factores que se asociaron al abandono temprano de la LM fueron los maternos: la existencia de conocimientos malos (RM 5.97, IC 95\% 1.67-20.67), no tener el antecedente de haber experimentado la LM en un hijo previo (RM 2.98, IC 95\% 1.66-5.36) y el plan materno de duración de la LM por tres meses o menos (RM 16.24, IC 95\% 5.37-49.12). El único factor laboral que mantuvo asociación con el abandono temprano de la LM fue el no contar con facilidades en el trabajo (RM 1.99, IC 95\% 1.12-3.56). De este análisis se excluyó a las siete madres que no planearon alimentar con leche materna, quienes en efecto no lo hicieron. Todas estas razones de momios están ajustadas además por la edad del niño al momento de la entrevista. Este modelo

\section{Cuadro III \\ Abandono temprano de la lactancia materna en RELACIÓN CON LOS FACTORES LABORALES. Ensenada, Baja California, México, 1998-1999}

\begin{tabular}{|c|c|c|}
\hline & Lactanci & materna \\
\hline & $\leq 3$ meses & $>3$ meses \\
\hline & & \\
\hline
\end{tabular}

O cupación

Maquiladora, obrera, cocinera

$\begin{array}{lllll}\text { intendente } & 54 & 48.2 & 73 & 47.7\end{array}$

Secretaria, vendedora, cajera,

$\begin{array}{llllll}\text { mesera } & 25 & 22.3 & 42 & 27.4 & \\ \text { Técnica, profesional } & 33 & 29.5 & 38 & 24.8 & 0.551\end{array}$

Duración de la jornada de trabajo $\quad 9.25$ (4-13) $\quad 9.00$ (3-12) 0.138 Jornada de trabajo

$\begin{array}{lllll}\text { Menor de ocho horas } & 21 & 18.8 & 29 & 19.0\end{array}$

$\begin{array}{llllll}0 \text { cho horas o más } & 91 & 81.2 & 124 & 81.0 & 0.967\end{array}$

Facilidades en el trabajo para la LM

\begin{tabular}{llllll} 
Sí & 41 & 36.6 & 81 & 52.9 & \\
\hline No & 71 & 63.4 & 72 & 47.1 & 0.008
\end{tabular}

Facilidades en el trabajo para la LM

\begin{tabular}{lrrrrr} 
Hora de lactancia & 35 & 85.4 & 59 & 72.8 & \\
\hline Lactancia en el trabajo & 2 & 4.9 & 15 & 18.5 & \\
\hline 0 tras & 4 & 9.8 & 7 & 8.6 & 0.121
\end{tabular}

$\mathrm{LM}=$ Lactancia materna

* $\chi^{2}$ para proporciones y $U$ de Mann-W hitney para medianas

* Lugar para la extracción de leche o licencia para ausentarse del trabajo por un mes más

salud pública de méxico / vol.45, no.4, julio-agosto de 2003

\section{Cuadro IV \\ Factores asociados al ABANDONo temprano DE LA LACTANCIA MATERNA.* Ensenada, Baja California, México, 1998-1999}

Factor RM* IC $95 \% \quad$ Valor $p$

Conocimientos sobre LM

\begin{tabular}{llll} 
Buenos & 1.00 & & \\
\hline Regulares & 3.17 & $1.00-10.06$ & 0.050 \\
\hline Malos & 5.97 & $1.67-20.67$ & 0.006
\end{tabular}

Antecedentes de LM

\begin{tabular}{lll} 
Sí & 1.00 & \\
\hline No & 2.98 & $1.66-5.36<0.001$
\end{tabular}

Plan de duración de la LM

\begin{tabular}{lrrr} 
Siete meses y más & 1.00 & & \\
\hline De cuatro a seis meses & 2.55 & $1.27-5.11$ & 0.008 \\
\hline De cero a tres meses & 16.24 & $5.37-49.12$ & $<0.001$ \\
\hline Sin plan o respuesta imprecisa & 3.87 & $1.09-13.75$ & 0.036
\end{tabular}

Facilidades para la LM en el trabajo

\begin{tabular}{llll} 
Sí & 1.00 & & \\
\hline No & 1.99 & $1.12-3.56$ & 0.019
\end{tabular}

RM* = Razones de momios ajustadas además por la edad del hijo al momento de la entrevista

IC = Intervalo de confianza

LM $=$ Lactancia materna

clasificó correctamente a 72.5\% de las madres; mostró una sensibilidad de $59.4 \%$, una especificidad de $81.6 \%$; el valor de la probabilidad de la $\chi^{2}$ de Hosmer-Lemeshow fue de 0.100 , lo que muestra un buen ajuste del modelo. Se probaron además algunas interacciones, como el tipo de conocimientos sobre LM con el antecedente de haber lactado a hijos previos, o con el tiempo planeado de duración de la LM, pero ninguna de ellas resultó significativa. Las variables potencialmente confusoras que se eliminaron fueron: las características demográficas de las madres, las características relacionadas con los servicios de salud, el tipo de ocupación y duración de la jornada de trabajo, el tipo de facilidades en el trabajo para la LM y el que los niños acudieran a guardería, ya que ninguna de éstas confundía las asociaciones antes descritas.

\section{Discusión}

Los resultados de este estudio sugieren que los factores maternos: conocimientos malos sobre LM, falta de experiencia en su práctica y el tiempo planeado de su 
duración, menor o igual a tres meses, junto con la carencia de facilidades en el trabajo para este tipo de alimentación, son factores que se asocian significativamente con el abandono temprano de la misma en madres trabajadoras de esta zona del país. El grupo de madres participantes en este estudio, todas derechohabientes del IMSS, fue homogéneo en sus principales características socioeconómicas y de escolaridad, y en la atención recibida en el servicio de salud durante su control prenatal y del parto. El tamaño de la muestra de este estudio nos permite contar con un nivel de confianza de $95 \%$ y con un poder de $80 \%$ en la evaluación de las asociaciones entre los diferentes factores y el abandono temprano de la LM; la condición anterior nos permite sustentar que las diferencias encontradas entre los dos grupos de madres son probablemente reales.

La decisión materna de alimentar al pecho y su duración tiene una influencia multifactorial; entre estos factores se destacan los socioeconómicos (estructura familiar, ingresos, red de apoyo familiar y de los servicios de salud), culturales y, en la mujer trabajadora, el plan de retorno a su trabajo, y las condiciones laborales (extensión de la jornada de trabajo y facilidades laborales para la LM). ${ }^{3,7,9-11,27}$ En las madres incluidas en este estudio destaca, por un lado, el haber tenido exposición a factores laborales de repercusión negativa para la LM, como son la duración excesiva de la jornada de trabajo, $, 23,28$ el predominio de las actividades físicas en su ocupación, y la carencia de facilidades para hacer más flexible su jornada, y el momento de reincorporación posparto al trabajo, sin arriesgar su estabilidad laboral. ${ }^{9,29,30}$ Por otro lado, se identifica un control prenatal oportuno aunado al contacto con el Programa Hospital Amigo del Niño y de la Madre como factores potencialmente positivos para la LM. Sin embargo, ambos tipos de factores se distribuyeron con una frecuencia semejante, tanto en las madres que la abandonaron tempranamente, como en aquellas que la prolongaron. Cabe preguntarse ¿qué condiciones condujeron a las madres a tomar decisiones diferentes?

El grado de compromiso de la madre con la LM, medido por el tiempo planeado de duración, parece ser el factor más importante para sostener la LMCE y prolongar los beneficios de la LM por medio de la lactancia mixta; es muy probable que el nivel de conocimientos sobre la LM y tener la experiencia de este tipo de alimentación en un hijo previo influya en la decisión de prolongarla; sin embargo, es factible que participen otros factores de orden psicosocial, principalmente aquellos relacionados con las redes de apoyo, ya que es muy llamativo que casi la mitad de las señoras en ambos grupos no buscaron ayuda con familiares o amigas en caso de dudas o problemas sobre la LM, y tampoco lo hicieron con el personal de salud. Esto puede estar revelando la existencia de grupo familiares o redes de apoyo mal estructuradas o disfuncionales, en su caso impermeables o muy selectivas al apoyo fuera de la familia nuclear, ${ }^{31}$ estos supuestos no pueden ser contestados en este estudio y podrían ser motivo de otra investigación.

A pesar de que los beneficios de la LM para la madre y su hijo son muchos, ${ }^{1,2}$ difundidos y reforzados principalmente durante la atención del parto en los Hospitales Amigos del Niño y de la Madre, ${ }^{17-19}$ condición a la que estuvieron expuestas todas las mujeres que participaron en este estudio, encontramos que la proporción de madres que la abandonaron temprano sigue siendo muy alta y que ésta conserva la tendencia a la baja en su duración global. Las principales acciones del programa en la asistencia a la madre para la LM, como son la asesoría para la primera tetada, la facilitación para su oportunidad y la proscripción de los biberones y los SLM se dieron en más de $80 \%$ de las madres de ambos grupos, y esto coincide con una intención de $95 \%$ o más de las madres en iniciar la LM. Sin embargo, posteriormente, aunque con diferente duración, ambos grupos abandonaron prematuramente la LMCE. Este comportamiento guarda semejanzas con los resultados de Pérez-Escamilla y Flores Huerta, ${ }^{11,15}$ ambos trabajos fueron realizados en áreas urbanas y sus hallazgos apoyan la repercusión del alojamiento conjunto sobre la permanencia de la LM durante el primer mes de vida posnatal. Por lo anterior, parece que las actividades de promoción de la LM que se realizan actualmente no son condición suficiente para influir en la decisión materna de amamantar por tiempo prolongado. Otro escenario de mayor duración que el hospitalario, y por tanto de mayor oportunidad, donde debe promoverse la LM, se ubica en el primer nivel de atención del sistema nacional de salud, durante la atención prenatal. La Norma Oficial Mexicana ${ }^{32}$ considera como estándar de una atención prenatal adecuada, su inicio lo más temprano posible durante el primer trimestre de la gestación y un mínimo de seis consultas durante el embarazo, en las cuales, junto con otras acciones relacionadas con la salud del feto y de la madre, se norma la promoción de la lactancia materna. Estas acciones no han sido evaluadas en su homogeneidad de contenidos para la educación, ni en su calidad. El mínimo de consultas prenatales se cumplió en más de $80 \%$ de las madres incluidas en esta investigación, sin que se pueda detectar un efecto benéfico de esta intervención potencialmente promotora de la LM en la duración de la misma. Puede uno preguntarse qué tan congruente es la información sobre LM 
que se transmite durante la atención prenatal y aquella que se da en los hospitales durante la atención del parto $y$, por lo mismo, se hace interesante investigar como una fuente de confusión para las madres el grado de continuidad que existe entre las actividades de promoción para la LM, que se realizan en unidades de primer contacto y la de los hospitales donde esta misma población acude a la atención del parto, ya que algunos estudios han demostrado un efecto mayor en la duración de la LM cuando las acciones de promoción de la misma se realizan, tanto durante el control prenatal, como durante el parto y en las consultas subsecuentes. ${ }^{33}$ Además, es necesario un mayor apoyo para la LM durante el control prenatal, ya que la decisión sobre la duración de ésta es tomada por la madre durante el embarazo. ${ }^{3,13}$

La incorporación de la mujer a la actividad económica es creciente y predominantemente fuera del hogar. En estas mujeres se ha observado que un factor importante para el abandono temprano de la LM es la separación de su hijo por periodos largos relacionados con su jornada de trabajo, ya que las madres que pueden tener cerca a sus hijos durante su trabajo, y por lo tanto pueden alimentarlos, logran mantener la LM por más tiempo. ${ }^{9}$ Por lo anterior, destaca entre los factores laborales la repercusión negativa de la ausencia de facilidades en el trabajo para la LM, condición que se suma a la reconocida inseguridad que muchas madres tienen sobre las cualidades nutritivas de su leche y sobre la suficiencia en la cantidad que pueden producir, $^{14,20}$ lo cual, según se ha reportado, influye sobre la decisión materna de introducir los SLM, e incluso de iniciar tempranamente la ablactación para asegurar de alguna manera la buena nutrición de su hijo. Esta inseguridad puede ser un reflejo de la falta de conocimientos sobre la LM, y parece congruente con la identificación de los malos conocimientos sobre la LM como un factor de riesgo para el abandono temprano de este tipo de alimentación. Parece necesario plantear intervenciones que aseguren a las madres saber más sobre lactancia materna durante la etapa prenatal y que sirvan para apoyar la permanencia de la LM en la etapa posnatal. Estas intervenciones pueden ser la implantación de programas específicos de promoción de la LM en el primer nivel de atención, durante el control prenatal, la integración de grupos de apoyo en el interior de los centros de trabajo, la adecuación de áreas específicas para la LM que permitan la extracción y almacenamiento de la leche materna, el cumplimiento, por parte del empleador, de las facilidades legales que debe brindar a las madres después del parto. Todo lo anterior, aunado al conocimiento por parte de las madres de sus derechos al respecto. ${ }^{24,34}$
Una limitación de este estudio es la aplicación posparto de un cuestionario en el que se pregunta el plan pre-parto de alimentar con leche materna y de su duración. Sin embargo, en favor de la validez de nuestros resultados, existe evidencia de que la mujer que trabaja fuera de su hogar planea en el pre-parto la forma como alimentará a su hijo, de acuerdo con el momento de reinicio de sus labores y de que sus conductas posparto son consistentes con las planeadas antes del nacimiento de su hijo., ${ }^{3,14,24,35}$ Por lo tanto, a pesar del momento de la aplicación del cuestionario, es probable que las respuestas realmente reflejen el plan pre-parto.

En este estudio se identifica tanto a un grupo de madres que abandonaron tempranamente la lactancia materna, debido a un plan de corta duración de la misma, subordinado al reinicio de su trabajo; además, también se identifica a otro grupo de madres que a pesar de tener un reinicio igual de sus actividades laborales, prolongó la LM, tal vez porque la manejaron con un nivel mayor de prioridad o al menos equivalente al del trabajo, postura que puede verse influida por los buenos conocimientos sobre la LM, una experiencia previa positiva con ella y por contar con facilidades en el trabajo para efectuarla.

\section{Referencias}

1. Goldman AS, Goldblum RM. D efense agents in milk. A defense agents in human milk. En: Jensen RG. Handbook of milk composition. San Diego Academic Press, 1995:727-738.

2. Secretaría de Salud. Dirección General de Salud Reproductiva. Ventajas de la lactancia materna. Manual de lactancia materna para el primer nivel de atención. México, DF: SSA, 1997:25-28.

3. Pérez-Escamilla R, Lutter Ch, Segall AM, RiveraA,Treviño-Siller S, Sanghvi T. Exclusive breast-feeding duration is associated with attitudinal, socioeconomic and biocultural determinants in three Latin American countries. J N utr 1995;125:2972-2984.

4. Pérez-Escamilla R. Patrones de lactancia natural en A mérica Latina y El Caribe. Bol 0 ficina Sanit Panam 1993;115:185-193.

5. Amador M, Silva L,Valdés-Lazo F. Tendencias de la lactancia materna en Cuba y las A méricas. Bol 0 ficina Sanit Panam 1994;116:204-211. 6. Santos-Torres I,Vázquez-G aribay E, N ápoles-Rodríguez F. Hábitos de lactancia materna en colonias marginadas de Guadalajara. Bol Med Hosp Infant Mex 1990;47:318-323.

7. Vega LMG , G onzález PGJ. Factores maternos asociados a la duración de la lactancia en áreas periféricas de Guadalajara, México. Bol 0 ficina Sanit Panam 1993;115(2):118-126.

8. Pérez-Escamilla R, D ewey KG . Epidemiología de la lactancia materna en zonas rurales y urbanas de México. Bol 0 ficina Sanit Panam 1993;114(5):399-405.

9.W inikoff $B, C$ astle $M$. The influence of maternal employment on infant feeding. En: Beverly W inikoff, Castle MA, H ight-Laukaran V, Ed. Feeding 
infants in four societies: $C$ auses and consequences of mothers' choices. W estport (et): G reenwood Press, 1988:121-145.

10. Pérez-Escamilla R, Segura-Millán S, Pollit E, D ewey KG. D eterminants of lactation performance across time in an urban population from Mexico. Soc Sci Med 1993;37:1069-1078.

11. Pérez-Escamilla R, Segura-Millán S, Pollit E, D ewey KG. Effect of the maternity ward system on the lactation success of low income urban Mexican women. Early Human Dev 1992;31:25-40.

12. O livas MG, Morales RM, C uéllar EJ. Lactancia materna poscesárea: seguimiento lácteo y neonatal. G inecol 0 bstet Mex 1990;58:251-255. 13. Lomelí GLE, Medina CL, Rodríguez JMG . Influencia de la educación prenatal en la decisión de lactar al seno materno. Rev Med Inst Mex Seguro Soc Mex 1996;34:473-476.

14. Segura-Millán S, D ewey KG, Pérez-Escamilla R. Factors associated with perceived insufficient milk in a low-income urban population in Mexico. J N utr 1994;124:202-212.

15. Flores-Huerta S, Cisneros-Silva I.Alojamiento conjunto madre-hijo y lactancia humana exclusiva. Salud Publica Mex 1997;39(2):110-116.

16. Long-D unlap K, Rivera D ommarco J, Rivera-Pasquel M, HérnandezA vila M, Lezana MA. Feeding patterns of Mexican infants recorded in the 1988 N ational Nutrition Survey. Salud Publica Mex 1995;37:120-129. 17. W orld Health 0 rganization/UN IC EF. Protecting, promotion and supporting breastfeeding:The special role of maternity service.A join W HO /UN IC EF statement. Int J Gynecol 0 bstet 1990;31:171-183. 18. Secretaría de Salud, Dirección General de Salud Reproductiva. Situación de la lactancia materna. En: Manual de lactancia materna para el primer nivel de atención. México, DF:SSA, 1997:7-20.

19. Fondo de las $\mathrm{N}$ aciones Unidas para la Infancia. La iniciativa hospital amigo del niño. ParteVII. Revaluación de hospitales amigos del niño: guía para desarrollar el proceso a nivel nacional. $N$ ueva York (N Y): FBH I Team N utrition Section,1998.

20. Margen S, Melnick V, N euhauser L, Ríos E. Infant feeding in Mexico. Emeryville (CA): PRIN TEAM 1991.

21. Kramer M, C halmers B, D onet E, Sevkovskaya Z, D zikovich I, Shapiro $S$ et al. Promotion of breastfeeding intervention trial (PRO BIT):A randomized trial in the Republic of Belarus. JAMA 2001;285:413-420.
22. Instituto $N$ acional de Estadística, Geografía e Informática. X II Censo General de Población yVivienda. México, 2000. Disponible en: http:// www.inegi.gob.mx

23. Carlson A, Faden $R, O^{\prime}$ Campo P, Brown H, Paige D. Maternal employment during the early postpartum period: Effects on initiation and continuation of breast-feeding. Pediatrics 1991;87:298-305.

24. Roe B,W hittington L, Fein S,TiesI M. Is there competition between breast-feeding and maternal employment? Demography 1999; 36: 157-171.

25. Hosmer D, Lemeshow S.Applied logistic regression. N ueva (N Y): John W iley Press, 1989.

26. Stata Corporation Stata Statistical Software: Release 6.0. College Station, Texas: Stata Corporation, 1996.

27. Ryan A, Martínez G. Breast-feeding and the working mother:A profile. Pediatrics 1989;83:524-531.

28. Fein S, Roe B.The effect of work status on initiation and duration of breast-feeding? Am J Public Health 1998;88:1042-1046.

29. Kurinuj N, Shiono P, Ezrine S, Rhoads G. D oes maternal employment affect breast-feeding. Am J Public Health 1989;79:1247-1250.

30. A uerbach K, G uss E. Maternal employment and breastfeeding. A study of 567 women's experiences.AJDC 1984;138:958-960.

31. Bronfman M. Como se vive se muere. Familia, redes sociales y muerte infantil. México, DF: UN AM, 2000:79-188.

32. Secretaría de Salud. N orma O ficial Mexicana N O M-007-SSA2-1993 para la atención de la mujer durante el embarazo, parto y puerperio y del recién nacido. C riterios y procedimientos para la prestación de servicio. México, DF: Diario O ficial de la Federación, 6 de enero de 1995.

33. Couto M, Bastos L, Tedstone A. Extending breastfeeding duration through primary care:A systematic review of prenatal and postnatal interventions. J Human Lact 2001;17:326-343.

34. Friesen $\mathrm{H}$, Vince J, Boas $\mathrm{P}, \mathrm{D}$ anya R. Protection of breastfeeding in Papua N ew Guinea. Bull W orld Health O rgan 1999;77:271-274. 35. N oble S,The ALSPAC Study Team. Maternal employment and the initiation of breastfeeding. Acta Paediatric 2001;90:423-428. 